\title{
Comment on: Disulfiram Induced Psychosis (Mohapatra and Rath, Clin Psychopharmacol Neurosci 2017;15:68-69)
}

\author{
Petros N. Karamanakos ${ }^{1}$, Eleftheria S. Panteli ${ }^{2}$ \\ ${ }^{1}$ Department of Neurosurgery, Olympion General Hospital and Rehabilitation Center, Patras, ${ }^{2}$ Department of Anesthesiology and Critical Care \\ Medicine, University Hospital of Patras, School of Medicine University of Patras, Patras, Greece
}

\section{TO THE EDITOR}

We read with great interest the recently published article by Mohapatra and Rath ${ }^{1)}$ regarding a case of psychosis after treatment with disulfiram. Because of our continuous research on disulfiram and its side effects, we find the issue very interesting and we would like to comment briefly on this report.

In the present manuscript the authors report a case of an alcoholic patient who developed psychosis one month after daily intake of $500 \mathrm{mg}$ of disulfiram. After discontinuation of the drug the patient improved and he completely recovered within two weeks. The main underlying mechanism suggested by the authors was the impairment of the metabolism of brain catecholamines, due to inhibition of dopamine-beta-hydroxylase (DBH), an enzyme that catalyzes the conversion of dopamine to noradrenaline in the catecholamine storage vesicles. ${ }^{2)}$ Implying that this is a dose-dependent phenomenon they recommended that disulfiram should be used at the lowest effective dose, possibly $250 \mathrm{mg}$ daily. ${ }^{1)}$

Disulfiram has been used for more than sixty years in the treatment of chronic alcoholism due to the unpleasant symptoms it provokes after ethanol intake. Classically, the alcohol intolerance produced by disulfiram has been attributed to the inhibition of the hepatic aldehyde deydrogenase (ALDH) and the subsequent accumulation of toxic amounts of acetaldehyde in the blood. In addition to provoking an unpleasant aversive reaction to ethanol, it is well known that disulfiram has also inherent toxicity toward the central and peripheral nervous system. ${ }^{3)}$ A key role in the neurotoxicity of disulfiram has been attributed

Received: February 6, 2017 / Accepted: February 9, 2017 Address for correspondence: Petros N. Karamanakos, MD, PhD Department of Neurosurgery, Olympion General Hospital and Rehabilitation Center, Volou and Milihou, 26443, Patras, Greece Tel: +30-6945548463, Fax: +30-2610464115 E-mail: pkaramanakos@olympion-sa.gr to the inhibition of $\mathrm{DBH}$, which was also suggested in the report of Mohapatra and Rath. ${ }^{1)}$

In a previous work of our laboratory team increasing doses of disulfiram were administered to Wistar rats and the activity of the hepatic ALDH along with the levels of brain catecholamines were determined. ${ }^{4)}$ According to our results, disulfiram affected the level of brain catecholamines at dose levels higher than those inhibiting the activity of ALDH, suggesting that ALDH was highly sensitive to the inhibitory effect of disulfiram, while on the contrary, the levels of brain catecholamines, and hence the activity of DBH, were more refractory to the action of this drug. As neurotoxicity of disulfiram is believed to be related with the produced changes in the levels of catecholamines through DBH inhibition, we concluded that in clinical practice, treatment of alcoholic patients with low disulfiram doses could retain the aversion effect toward ethanol, with a reduced risk of neurotoxicity (e.g., psychosis), underlining the dose-dependent property of this side effect.

In conclusion, the article by Mohapatra and Rath ${ }^{1)}$ is really interesting because it adds one more case of psychosis after treatment with disulfiram. However, their recommendation for daily use of no more than $250 \mathrm{mg}$ disulfiram is somehow arbitrary. Consequently, we believe that the authors should refer our work in their paper in order to strengthen their assumption regarding the dose-dependent nature of disulfiram-induced psychosis and highlight that there is a safety margin in the dosage of disulfiram between therapeutic and toxic effects.

\section{REFERENCES}

1. Mohapatra S, Rath NR. Disulfiram Induced Psychosis. Clin Psychopharmacol Neurosci 2017;15:68-69.

2. Vaccari A, Saba PL, Ruiu S, Collu M, Devoto P. Disulfiram and diethyldithiocarbamate intoxication affects the storage and release of striatal dopamine. Toxicol Appl Pharmacol 1996;139:102-108.

(C) This is an Open-Access article distributed under the terms of the Creative Commons Attribution Non-Commercial License (http://creativecommons.org/licenses/by-nc/4.0) which permits unrestricted non-commercial use, distribution, and reproduction in any medium, provided the original work is properly cited. 
3. Haley TJ. Disulfiram (tetraethylthioperoxydicarbonic diamide): a reappraisal of its toxicity and therapeutic application. Drug Metab Rev 1979;9:319-335.

4. Karamanakos PN, Pappas P, Stephanou P, Marselos M.
Differentiation of disulfiram effects on central catecholamines and hepatic ethanol metabolism. Pharmacol Toxicol 2001;88:106-110. 